\title{
Secrecy analysis of cognitive radio network with MS-GSC/MRC scheme
}

\author{
Shilpa Thakur ${ }^{*}$ (1) and Ajay Singh ${ }^{2}$
}

\begin{abstract}
We propose and analyze minimum selection-generalized selection combining (MS-GSC) at secondary receiver (SR) with maximal ratio combining at eavesdropper (ER) to enhance data security at physical layer. We consider an underlay cognitive radio network (CRN) where SR and ER are equipped with multiple antennas, and secondary transmitter (ST) has single antenna with a primary user. Passive eavesdropping is also taken into account.This work is aimed to find the effect of MS-GSC diversity technique on secrecy outage probability (SOP). We derive a closed-form expressions for the exact and asymptotic SOP. Our results show a positive impact on SOP with an increase in diversity branches and also reveal the effect of a primary user on secondary network.
\end{abstract}

Keywords: Cognitive radio network, Minimum selection-generalized selection combining (MS-GSC), Outage probability, Physical layer security

\section{Introduction}

In wireless communication systems, an eavesdropper intercepts transmission due to the broadcast nature of wireless links. So, the security of data transmission in these networks is becoming more critical than ever $[1,2]$. Traditionally, cryptographic techniques are used to secure data at the upper layer of protocol stack using public and private key variations. In underlay cognitive radio networks (CRN), the primary users (PU) and the secondary users (SU) transmit concurrently in the same band of frequency $[3,4]$. The protection and security of the broadcast channel in such complex environments against eavesdropping is a very difficult task. The conventional cryptographic authentication become very expensive and less effective because of the open nature of these broadcasting channels $[5,6]$. Therefore, research efforts have been devoted to physical layer security, which exploits the characteristics of wireless channel (e.g., thermal noise and fading) to secure the communication at physical layer [7]. The fundamental concept of physical layer security is to enhance the gain of legitimate receiver's main channel in comparison to the eavesdropper's channel to attain perfect secrecy. With the advancement in multiple antenna

*Correspondence: shilpathakur_1987@rediffmail.com

${ }^{1}$ Department of Electronics and Communication Engineering, National Institute of Technology, Hamirpur, India

Full list of author information is available at the end of the article techniques, security improvement in wiretap CRN channels has addressed from information-theoretic perspective [8-11], where the secondary transmitter (ST), the secondary user receiver (SR), and the eavesdropper (ER) all are equipped with multiple antennas.

In the last few years, lots of work had been done to enhance the security of physical layer in CRN. In $[12,13]$, secrecy performance of single input multiple output (SIMO) CRN with maximal ratio combining (MRC) diversity technique was studied. Secrecy outage probability (SOP) for transmit antenna selection (TAS)/MRC in multiple input multiple output (MIMO) CRN has been investigated in [14], and an underlay MIMO CRN with a pair of primary nodes and secondary nodes and an eavesdropper was considered in [15] where the secondary transmitter was powered by the renewable energy harvested from the primary transmitter in order to improve both energy efficiency and spectral efficiency. SOP of an underlay cognitive decode-and-forward relay network over independent but not necessarily identical distributed (i.n.i.d) Nakagami-m fading channels was investigated, and optimal relay selection (ORS) and suboptimal relay selection (SRS) schemes, and multiple relay combining scheme were considered in [16]. In [17], a hybrid visible light communication radio frequency (RF) system with legitimate receiver and an eavesdropper was considered where legitimate receiver can harvest energy from the light emitted 
by light-emitting diodes, and exact and asymptotic SOP was derived by using stochastic geometry method. The closed-form expressions for SOP and non-zero secrecy capacity for underlay CR unit over Nakagami-m fading were investigated in [18], and the secrecy outage performance of PU system in the presence of the eavesdropping and interfering of SU was analyzed in [19]. Generalizedselection combining (GSC) is a hybrid technique that overcomes the limitation of MRC and selection combining (SC). In [20-22], a detailed study has been done on GSC. GSC uses a fixed number of the best branches $\left(M_{c}\right)$ of all available ones $(M)$. In GSC, there is no need of process all the paths that reduce the hardware complexity of receiver, but all MRC branches remain active during the reception of the data, which increases consumption of computational power [20]. The secrecy analysis for SIMO wiretap CRN with GSC over Nakagami-m fading channels was done in [22]. TAS with GSC over Rayleigh fading and Nakagami-m fading channels were applied in [23-26]. In [26], TAS/GSC for cognitive decode-andforward relaying in Nakagami- $\mathrm{m}$ fading channels was considered and closed-form expression for ergodic capacity was derived. The power saving implementation of GSC called minimum selection GSC (MS-GSC) had proposed in [27].

In this paper, we consider a communication scenario where MS-GSC diversity combining technique is adopted by the SR considering the complexity and energy dissipation, and in order to maximize, its instantaneous SNR at ER MRC technique is applied. In fact, MS-GSC is a more general diversity combing than GSC. The basic idea of MS-GSC is that the minimum number of diversity branches are selected such that their combined signal-tonoise ratio (SNR), and not the individual branch SNR, is above a given threshold [28].

Our contributions are as follows:

1. We examine the secrecy performance of an underlay CRN with MS-GSC applied at legitimate receiver and MRC at eavesdropper over Rayleigh fading environment and derived closed-form expression for SOP.

2. In [2], interference power constraint was considered at primary user (PU). Both SR and ER were equipped with multiple antennas, and selection combining (SC) was applied. SC select only one antenna with the highest SNR among available ones which neglect diversity phenomena. In comparison to [2], interference power constraint, more generalized, and computational power saving system is considered in this paper.

3. In [12], MRC scheme was used over Rayleigh fading channels at both secondary receiver SR and eavesdropper. We apply MS-GSC at SR and MRC at eavesdropper and SC $\left(M_{C}=1\right), \operatorname{MRC}\left(M_{C}=M\right)$, and GSC $\left(M_{C} \leq M\right)$ are the special cases of MS-GSC.

The organization of the remaining paper is as follows: Section 2 explains the proposed system model. Furthermore, the working of MS-GSC is also explained in Section 2. Secrecy outage probability is calculated in Section 3. Section 4 gives the interpretation of numerical result.

\section{System model}

Here, we consider an underlay wiretap CRN composed of a primary user (PU), secondary user transmitter as Alice, legitimate receiver as $B$, and an eavesdropper as ER. The primary user and Alice are consist of a single antenna whereas $B$ and the ER are assumed to have multiple antennas $M$. Here, we assume that the confidential messages are being transmitted from Alice to $B$ in the presence of ER, and the ER want to listen their communication. In order to have reliable communication, the interference power at $P U$ from Alice should be less than the peak interference power threshold.

The primary and secondary channels are experiencing i.i.d Rayleigh fading where channel gains of the main channel $\left\{h_{B}\right\}_{t=1}^{M}$, eavesdropper's channel $\left\{h_{E s}\right\}_{s=1}^{M}$, and primary channel $h_{p}$ are complex Gaussian random variables with zero mean and variances $\Omega_{1}, \Omega_{2}$, and $\Omega_{0}$, respectively. We are considering passive eavesdropping, i.e., the channel state information (CSI) of the eavesdropper is also available at Alice. The channel gain and variance of the primary users are $h_{p}$ and $\Omega_{0}$, respectively. The main channels (Alice to $B$ ) and eavesdropper channels (Alice to ER) are independent of each other. Here, the MS-GSC diversity scheme is applied at $B$, and MRC diversity technique is applied at $E R$. In this paper, we assume that the global CSI of the main links, the eavesdropper's links, and primary link is available for evaluating the secrecy rate in the information receiver, which is a common assumption in the literature on physical layer security. Information on the PU's channels can be obtained for the cases in which the PUs are cooperative in the network and their transmissions can be monitored. This is applicable for those networks that combining multicast and unicast transmissions, in which terminals play dual roles as legitimate receivers for some signals and eavesdroppers for others. In practice, this information can be generated by using reverse training, where the primary user transmits training signal to Alice such that by invoking the principle of reciprocity, Alice can estimate the primary users CSI.

The interference received at secondary network from primary network is considered to be a complex Gaussian random variable under an assumption that the primary signal may be generated by the random Gaussian codebook. Moreover, the thermal noise at secondary nodes 
is also complex Gaussian distributed. Thus, the interference plus noise at secondary nodes ( $B$ and eavesdroppers) can be modeled as a complex Gaussian random variable with zero mean and variance $N_{0}$. It indicates that the influence of primary interference at secondary nodes is already assumed in the variance $N_{0}$. Thus, the effect of interference is adjusted in the noise statistics at $B$ and $E R$ $[29,30]$. The instantaneous SNR of the main channels and eavesdropper's channel is given by

$$
\Psi_{M}=\max _{t=1 \ldots . . M} \frac{P_{a v}}{N_{0}}\left|h_{B_{t}}\right|^{2}, \Psi_{E}=\max _{s=1 \ldots . M} \frac{P_{a v}}{N_{0}}\left|h_{E_{s}}\right|^{2}
$$

where $P_{a v}$ is Alice's transmitted power.

\subsection{Working of MS-GSC diversity technique}

The receiver with MS-GSC diversity schemes chooses the minimum number of best available antennas in such a way that the combined SNR $\Psi_{\text {com }}$ is always more than the threshold SNR $\Psi_{t}$ [22]. Mathematically, the working of MS-GSC can be summarized as

$$
\Psi_{\mathrm{com}}= \begin{cases}\Psi(1) & \text { iff } \Psi_{1} \geq \Psi_{t} ; \\ \Psi(1)+\Psi(2) & \text { iff } \Psi(1)<\Psi_{t} \& \Psi(1)+\Psi(2) \geq \Psi_{t} ; \\ & \vdots \\ \sum_{k=1}^{r} \Psi(k) & \text { iff } \sum_{k=1}^{r-1} \Psi(k)<\Psi_{t} \& \sum_{k=1}^{r} \Psi(k) \geq \Psi_{t} ; \\ & \vdots \\ \sum_{k=1}^{M_{c}} \Psi(k) & \text { iff } \sum_{k=1}^{r-1} \Psi(k)<\Psi_{t} .\end{cases}
$$

where $\psi_{1}, \psi_{2}, \ldots . . . \psi_{k}$ are the SNR of path 1 , path $2 \ldots . .$. path $k$, respectively.

GSC uses $M_{c}$ antennas among available $M$ antennas such that $M_{c}<M$. In comparison to GSC, MS-GSC needs less computing power, and on average, less number of MRC branches is active during the reception of data which save processing power. Let $N_{b}$ be the active number of MRC branches, which takes the value from 1 to $M_{c}$. $N_{b}=1$ if $\Psi_{1}>\Psi_{t}, N_{b}=r, 2 \leq r \leq M_{c}-1$ if and only if $\sum_{k=1}^{r-1} \Psi_{k}<\Psi_{t}$ and $\sum_{k=1}^{r} \Psi_{k} \geq \Psi_{t}$, and $N_{b}=M_{c}$ if $\sum_{k=1}^{M_{c}-1} \Psi_{k}<\Psi_{t}$

\section{Secrecy outage probability}

The secrecy capacity analysis can help us to determine how secure a CRN is and whether we need more security mechanisms to protect against the potential attacks in the CRNs. The maximum achievable rate is named as secrecy capacity and given by ([2], Eq.2). The secrecy capacity of CRN consists an antenna at Alice and multiple antennas at $B$ and $E R$ can be defined as,

$$
S_{c}= \begin{cases}M_{c}-E_{e}=\log _{2}\left(\frac{1+\Psi_{M}}{1+\Psi_{E}}\right) & \text { if } \Psi_{M}>\Psi_{E}, \\ 0 & \text { if } \Psi_{M} \leq \Psi_{E},\end{cases}
$$

where $M_{c}=\log _{2}\left(1+\Psi_{M}\right)$ is the capacity of channels between Alice and $B$ and $E_{e}=\log _{2}\left(1+\Psi_{E}\right)$ is the capacity of channels between Alice and ER. $S_{c}$ in (4) can be rewritten as

$$
S_{c}=\log _{2}\left(\frac{1+\Psi_{M}}{1+\Psi_{E}}\right)<R_{s}
$$

which is analogous to

$$
\epsilon\left(\Psi_{E}\right)=2^{R s}\left(1+\Psi_{E}\right)-1>\Psi_{M}
$$

In passive eavesdropping, excellent secrecy is possible if and only if $R_{S} \leq S_{c}$; otherwise, information-theoretic security is compromised. SOP is a probability that secrecy capacity $S_{c}$ falls under the output threshold $R_{S}$ [2] and is given as

$$
\begin{array}{r}
P_{\text {out }}=P_{r}\left(S_{C}<R_{S}\right)=P_{r}\left(\Psi_{M} \leq \Psi_{E}\right) \\
+P_{r}\left(\Psi_{M}>\Psi_{E}\right) P_{r}\left(S_{c}<R_{S} \mid \Psi_{M}>\Psi_{E}\right)
\end{array}
$$

which can be simplified to (7)

$$
P_{\text {out }}=\int_{0}^{\infty} \int_{0}^{\infty} F_{\Psi_{M} \mid\{Y=y\}}\left(\in\left(\Psi_{E}\right)\right) f_{\Psi_{E} \mid\{Y=y\}}\left(\Psi_{E}\right) f_{Y}(y) d_{\Psi_{E}} d y
$$

where $Y=\left|h_{P}\right|^{2}$ is the channel gain from Alice to PU, $f_{Y}(y)$ is the probability density function (PDF) of $Y, f_{\Psi_{E} \mid(Y=y)}$ is the PDF of $\Psi_{E}$ conditioned on $Y$, and $F_{\Psi_{M} \mid Y=y}\left(\epsilon\left(\Psi_{E}\right)\right)$ is the cumulative distribution function (CDF) of $\Psi_{M}$ conditioned on $Y$.

In underlay cognitive radio transmission, for reliable communication, Alice's transmitted power $P_{\text {av }}$ should be less than the peak interference power threshold. So, Alice is a power-limited transmitter with a maximum transmit power which is $P_{T}$. The transmitted power of Alice is constrained by $P_{T}$ at Alice and peak interference power $P_{I}$ at the primary user and given by

$$
P_{\mathrm{av}}=\min \left(\frac{P_{I}}{h_{p}}, P_{T}\right)
$$

Based on (8) instantaneous SNR at secondary receiver, $B$ and $E R$ are expressed as

$\psi_{M}=\min \left(\frac{\psi_{p}}{Y}, \psi_{0}\right) X_{M} \quad \psi_{E}=\min \left(\frac{\psi_{p}}{Y}, \psi_{0}\right) X_{E}$

where $\psi_{p}=\frac{P_{I}}{N_{0}}, \psi_{0}=\frac{P_{T}}{N_{0}}, X_{M}=\max _{t=1 \ldots M}\left|h_{B_{t}}\right|^{2}$ and $X_{E}=\max _{s=1 \ldots . M}\left|h_{E_{s}}\right|^{2}$.

For ease of interpretation, we have $\Psi_{1}=\Omega_{1} \Psi_{0}=\psi_{P} \frac{\Omega_{1}}{\sigma}$ be the average SNR of the main channel, $\Psi_{2}=\Omega_{2} \Psi_{0}=$ $\psi_{P} \frac{\Omega_{2}}{\sigma}$ be the average SNR of the ED's channel, and $\sigma=$ $\frac{P_{I}}{P_{T}}$. The CDF for MS-GSC scheme for Rayleigh fading is given by ([22], Eq. 24) 


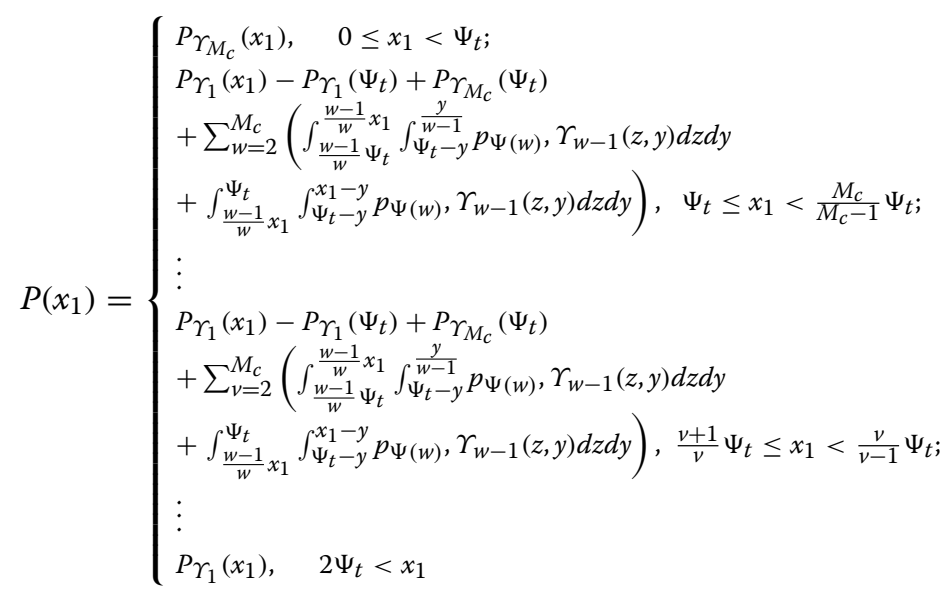

The CDF $P_{\Psi_{w}}($.$) is given by ([22], Eq. 17)$

$$
\begin{aligned}
\operatorname{P\Upsilon }_{w}\left(x_{1}\right)= & \frac{M !}{(M-w) ! w !}\left\{1-e^{\frac{-x_{1}}{\Psi_{1}}} \sum_{k=0}^{w-1} \frac{1}{k !}\left(\frac{x_{1}}{\Psi_{1}}\right)^{k}\right. \\
& +\sum_{v=1}^{M-w}(-1)^{w+v-1} \frac{(M-w) !}{(M-w-v) ! v !}\left(\frac{w}{v}\right)^{w-1} \\
& \times\left[\left(1+\frac{v}{w}\right)^{-1}\left[1-e^{-\left(1+\frac{v}{w}\right) \frac{x_{1}}{\Psi_{1}}}\right]\right. \\
& \left.\left.-\sum_{m=0}^{w-2}\left(\frac{-v}{w}\right)^{m}\left(1-e^{\frac{-x_{1}}{\Psi_{1}}} \sum_{k=0}^{m} \frac{1}{k !}\left(\frac{x_{1}}{\Psi_{1}}\right)^{k}\right)\right]\right\}
\end{aligned}
$$

where $x_{1}=2^{R s}(1+x)-1$ and the PDF of MRC $\left[p_{\Upsilon_{M}}().\right]$ is given by

$$
p_{\Psi_{M}}(x)=e^{\frac{-x}{\Psi_{2}}} \frac{x^{M-1}}{\Psi_{2}^{M}(M-1) !}
$$

where $\Psi_{2}$ is the average SNR of the ER's channels. The PDF of $Y$ is given by ([2], Eq. 22)

$$
f_{R}(r)=\sum_{h=0}^{N-1} \frac{N}{\Omega_{0}}(-1)^{h} e^{\frac{-(h+1) r}{\Omega_{0}}}, y \geq 0
$$

for $N=1$

$$
f_{R}(r)=\frac{1}{\Omega_{0}} e^{\frac{-(r)}{\Omega_{0}}}, \quad r \geq 0
$$

Using all the above mentioned equations in (7), SOP for proposed system model is given by (15).

$$
P_{\text {out }}= \begin{cases}P_{\text {out }_{A}} & 0 \leq x_{1}<\Psi_{t} \\ P_{\text {out }_{B}} & \Psi_{t} \leq x_{1}<\frac{M_{c}}{M_{c}-1} \Psi_{t} \\ \vdots & \\ P_{\text {out }_{C}} \frac{v+1}{v} \Psi_{t} \leq x_{1}<\frac{v}{v-1} \Psi_{t} \\ \vdots \\ P_{\text {out }_{D}} 2 \Psi_{t}<x_{1}\end{cases}
$$

The detail expansion of the above equation is as follows:

$$
\begin{aligned}
& p_{\text {out }_{A}}=\frac{M !}{M_{C} !\left(M-M_{C}\right) !}\left\{( 1 - e ^ { \frac { - \sigma } { \Omega _ { 0 } } } ) \left(1-\sum_{k=0}^{M_{c}-1} \sum_{n=0}^{k} \mu_{1}\right.\right. \\
& e^{\left(\frac{-\left(2^{R s}-1\right)}{\psi_{1}}\right)}+\sum_{v=1}^{M-M_{c}} C_{2} C_{3}-\sum_{v=1}^{M-M_{c} M_{c}-2} \sum_{m=0}^{M} C_{2} C_{4}-\sum_{v=1}^{M-M_{c}} C_{2} C_{4} \beta_{1} \\
& e^{-\left(\frac{\left(2^{R s}-1\right)\left(1+\frac{V}{M_{c}}\right)}{\psi_{1}}\right)}+\sum_{v=1}^{M-M_{c}} \sum_{m=0}^{M_{c}-2} \sum_{k=0}^{m} \sum_{n=0}^{k} C_{2} C_{4} \mu_{1} \\
& \left.e^{-\left(\frac{2^{R S}-1}{\psi_{1}}\right)}\right)+e^{\frac{-\sigma}{\Omega_{0}}}-\sum_{k=0}^{M_{c}-1} \sum_{n=0}^{k} \sum_{p=0}^{k-n} Q_{1} Q_{2} e^{-\sigma\left(\frac{2 R s-1}{\sigma \psi_{1}}+\frac{1}{\Omega_{0}}\right)} \\
& +\sum_{v=1}^{M-M_{c}} C_{2} C_{3} e^{-\left(\frac{\sigma}{\Omega_{0}}\right)}-\sum_{v=1}^{M-M_{c}} C_{2} C_{3} \beta_{2} \\
& e^{-\sigma\left(\left(1+\frac{M}{M_{c}}\right)\left(\frac{2^{R S}-1}{\sigma \gamma_{1}}\right)+\frac{1}{\Omega_{0}}\right)}-\sum_{\nu=1}^{M-M_{c} M_{c}-2} \sum_{m=0} C_{2} C_{4} e^{-\left(\frac{\sigma}{\Omega_{0}}\right)} \\
& \left.+\sum_{v=1}^{M-M_{c}} \sum_{m=0}^{M_{c}-2} \sum_{k=0}^{m} \sum_{n=0}^{k} \sum_{p=0}^{k-n} C_{2} C_{4} Q_{1} Q_{2} e^{-\sigma\left(\frac{2^{R s}-1}{\sigma \Psi_{1}}+\frac{1}{\Omega_{0}}\right)}\right\}
\end{aligned}
$$

where

$$
\begin{aligned}
\mu_{1}= & \frac{1}{(M-1) ! k ! \Psi_{1}{ }^{k}}\left(\begin{array}{l}
k \\
n
\end{array}\right)\left(2^{R s}-1\right)^{k-n} \frac{\left(2^{R s}\right)^{n}}{\left(\Psi_{2}\right)^{M}} \frac{(n+M-1) !}{\left(2^{R s}+\frac{1}{\Psi_{1}}\right)^{n+M}} \\
C_{2}= & \sum_{v=1}^{M-M_{c}}(-1)^{M_{c}+v-1} \frac{\left(M-M_{c}\right) !}{\left(M-M_{c}-v\right) ! v !}\left(\frac{M_{c}}{v}\right)^{M_{c}-1} \\
C_{3}= & \left(1+\frac{v}{M_{c}}\right)^{-1}, C_{4}=\left(\frac{-v}{M_{c}}\right)^{m} \\
\beta_{1}= & \frac{1}{\Psi_{2} M\left(\frac{\left(1+\frac{v}{M_{c}}\right) 2^{R s}}{\Psi_{1}}+\frac{1}{\Psi_{2}}\right)^{M}} \\
Q_{1}= & \frac{1}{k !\left(\sigma \Psi_{1}\right)^{k}}\left(2^{R s}-1\right)^{k-n}\left(2^{R s}\right)^{n}\left(\begin{array}{l}
k \\
n
\end{array}\right) \frac{(n+M-1) !}{\left(\frac{2^{R s}}{\sigma \Psi_{1}}+\frac{1}{\Psi_{2}}\right)^{n+M}} \\
Q_{2}= & \frac{(\sigma)^{p}(k-n) !}{\left(\sigma \Psi_{2}\right)^{M}(M-1) ! p !\left(\frac{2^{R s}-1}{\sigma \Psi_{1}}+\frac{1}{\Omega_{0}}\right)^{k-n-p+1}} \\
\beta_{2}= & \frac{1}{\left(\sigma \Psi_{2}\right)^{M}\left(\frac{\left(1+\frac{l}{M_{c}}\right)^{R s}}{\sigma \Psi_{1}}+\frac{1}{\sigma \Psi_{2}}\right)^{M}} \\
& \times \frac{1}{\left(\left(1+\frac{M}{M_{c}}\right)\left(\frac{2^{R s}-1}{\sigma \Psi_{1}}\right)+\left(\frac{1}{\Omega_{0}}\right)\right)}
\end{aligned}
$$




$$
\begin{aligned}
& P_{\text {out }_{B}}=P_{\text {out }_{D}}-P_{\text {out }_{1}}\left(\Psi_{t}\right)+P_{\text {out }_{M_{C}}}\left(\Psi_{t}\right)+\sum_{w=2}^{M_{C}} P_{\text {out }_{M}} \\
& P_{\text {out }_{1}}\left(\Psi_{t}\right)=\sum_{v=0}^{M}\left(\begin{array}{c}
M \\
v
\end{array}\right)(-1)^{v}\left[e^{\frac{-v \psi_{t}}{\psi_{1}}}\left(1-e^{\frac{-\sigma}{\Omega_{0}}}\right)+\frac{e^{-\sigma\left(\frac{v \psi_{t}}{\psi_{1}}+\frac{\sigma}{\Omega_{0}}\right)}}{\left(\frac{v \psi_{t}}{\sigma \psi_{1}}+\frac{1}{\Omega_{0}}\right)}\right] \\
& P_{\text {out }_{M}}=I_{1}+1_{2} \\
& I_{1}=\int_{0}^{\sigma} \int_{0}^{\infty}\left[\int_{\frac{w-1}{w} \Psi_{t}}^{\frac{w-1}{w} x_{1}} \int_{\Psi_{t-y}}^{\frac{y}{w-1}} p_{\Psi(w)}, \Upsilon_{w-1}(z, y) d z d y+\int_{\frac{w-1}{w} x_{1}}^{\Psi_{t}} \int_{\Psi_{t-y}}^{x_{1}-y} p_{\Psi(w)}, \Upsilon_{w-1}(z, y) d z d y\right] e^{\frac{-x}{\Psi_{2}}} \frac{x^{M-1}}{\Psi_{2}^{M}(M-1) !} \frac{1}{\Omega_{0}} e^{-\frac{r}{\Omega_{0}}} d x d r \\
& I_{2}=\int_{\sigma}^{\infty} \int_{0}^{\infty}\left[\int_{\frac{w-1}{w} \Psi_{t}}^{\frac{w-1}{w} x_{1}} \int_{\Psi_{t}-y}^{\frac{y}{w-1}} p_{\Psi(w)}, \Upsilon_{w-1}(z, y) d z d y+\int_{\frac{w-1}{w} x_{1}}^{\Psi_{t}} \int_{\Psi_{t}-y}^{x_{1}-y} p_{\Psi(w)}, \Upsilon_{w-1}(z, y) d z d y\right] e^{\frac{-x}{\Psi_{2}}} \frac{x^{M-1}}{\Psi_{2}^{M}(M-1) !} \frac{1}{\Omega_{0}} e^{-\frac{r}{\Omega_{0}}} d x d r \\
& P_{\text {out }_{M_{c}}}\left(\Psi_{t}\right)=\left(1-e^{\frac{-\sigma}{\Omega_{0}}}\right) \frac{M !}{\left(M-M_{c}\right) ! M_{c} !}\left\{1-e^{\frac{-\Psi_{t}}{\Psi_{1}}} \sum_{k=0}^{M_{\mathcal{c}}-1} \frac{1}{k !}\left(\frac{\Psi_{t}}{\Psi_{1}}\right)^{k}+\sum_{v=1}^{M-M_{c}} C_{2}\left[C_{3}\left(1-e^{-\left(1+\frac{v}{M_{c}}\right) \frac{\Psi_{t}}{\Psi_{1}}}\right)-\sum_{m=0}^{M_{c}-2} C_{4}\left(1-e^{\frac{-\Psi_{t}}{\Psi_{1}}} \sum_{k=0}^{m} \frac{1}{k !}\left(\frac{\Psi_{t}}{\Psi_{1}}\right)^{k}\right)\right]\right\} \\
& +\frac{M !}{\left(M-M_{c}\right) ! M_{c} !}\left[e^{\frac{-\sigma}{\Omega_{0}}}\left(1+\sum_{v=1}^{M-M_{c}} C_{2} C_{3}-\sum_{v=1}^{M-M_{c}} \sum_{m=0}^{M_{c}-2} C_{2} C_{4}\right)\right. \\
& \left.-\frac{1}{\Omega_{0}} \sum_{k=0}^{M_{c}-1} \sum_{l=0}^{k} \frac{1}{k !}\left(\frac{\psi_{t}}{\sigma \psi_{1}}\right)^{k} e^{-\left(\frac{\psi_{t}}{\psi_{1}}+\frac{\sigma}{\Omega_{0}}\right)} \frac{k !}{l !} \frac{(\sigma)^{l}}{\left(\frac{\psi_{t}}{\sigma \psi_{1}}+\frac{1}{\Omega_{0}}\right)^{k-l+1}}\left(1-\sum_{v=1}^{M-M_{c} M_{c}-2} \sum_{m=0} C_{2} C_{4}\right)-\frac{1}{\Omega_{0}} \sum_{v=1}^{M-M_{c}} C_{2} C_{3} \frac{e^{-\left(\frac{\psi_{t}}{C_{3} \sigma \psi_{1}}+\frac{\sigma}{\Omega_{0}}\right)}}{\frac{\psi_{t}}{C_{3} \sigma \psi_{1}}+\frac{\sigma}{\Omega_{0}}}\right]
\end{aligned}
$$

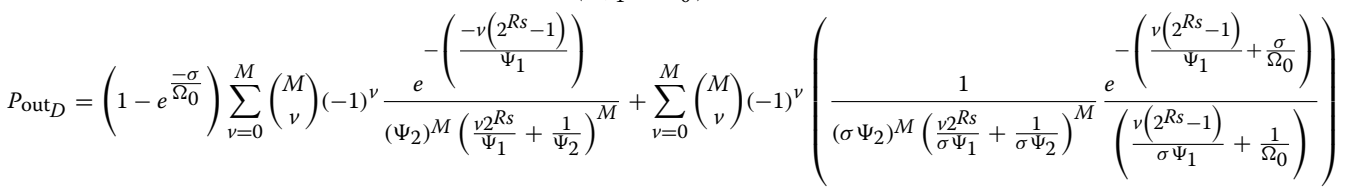

$$
\begin{aligned}
& P_{\text {out }_{C}}=P_{\text {out }_{D}}-P_{\text {out }_{1}}\left(\Psi_{t}\right)+P_{\text {out }_{v}}\left(\Psi_{t}\right)+\sum_{w=2}^{v} P_{\text {out }_{M}} \\
& P_{\text {out }_{v}}\left(\Psi_{t}\right)=\left(1-e^{\frac{-\sigma}{\Omega_{0}}}\right) \frac{M !}{(M-v) ! v !}\left\{1-e^{\frac{-\Psi_{t}}{\Psi_{1}}} \sum_{k=0}^{v-1} \frac{1}{k !}\left(\frac{\Psi_{t}}{\Psi_{1}}\right)^{k}+\sum_{v=1}^{M-v}(-1)^{2 v-1} \frac{(M-v) !}{(M-v-v) ! v !} \times\left[\frac{1}{2}\left[1-e^{-\frac{2 \Psi_{t}}{\Psi_{1}}}\right]\right.\right. \\
& \left.\left.-\sum_{m=0}^{v-2}(-1)^{m}\left(1-e^{\frac{-\Psi_{t}}{\Psi_{1}}} \sum_{k=0}^{m} \frac{1}{k !}\left(\frac{\Psi_{t}}{\Psi_{1}}\right)^{k}\right)\right]\right\}+\frac{M !}{(M-v) ! v}\left[e^{\frac{-\sigma}{\Omega_{0}}}\left(1+\sum_{v=1}^{M-v} \frac{1}{2} K_{1}-\sum_{v=1}^{M-v} \sum_{m=0}^{v-2} K_{1}(-1)^{m}\right)\right. \\
& \left.-\frac{1}{\Omega_{0}} \sum_{k=0}^{v-1} \sum_{l=0}^{k} \frac{1}{k !}\left(\frac{\psi_{t}}{\sigma \psi_{1}}\right)^{k} e^{-\left(\frac{\psi_{t}}{\psi_{1}}+\frac{\sigma}{\Omega_{0}}\right)} \frac{k !}{l !} \frac{(\sigma)^{l}}{\left[\frac{\psi_{t}}{\psi_{1} \sigma}+\frac{1}{\Omega_{0}}\right]^{k-l+1}}\left(1-\sum_{v=1}^{M-v} \sum_{m=0}^{v-2} K_{1}(-1)^{m}\right)-\frac{1}{\Omega_{0}} \sum_{v=1}^{M-v} \frac{1}{2} K_{1} \frac{e^{-\left(\frac{2 \psi_{t}}{\psi_{1}}+\frac{\sigma}{\Omega_{0}}\right)}}{\left(\frac{2 \psi_{t}}{\sigma \psi_{1}}+\frac{1}{\Omega_{0}}\right)}\right] \\
& \text { where } K_{1}=(-1)^{2 v-1}\left(\begin{array}{c}
M-v \\
v
\end{array}\right) \\
& N_{b}=\sum_{n=1}^{M_{c}} n \operatorname{Pr}\left[N_{b}=n\right] \sum_{n=1}^{M_{c}-1} p_{\Upsilon_{n}}\left(\Psi_{t}\right)
\end{aligned}
$$
and single antenna Alice in Rayleigh fading environment.

2. $P_{\text {out }}$ is the SOP corresponding to SC technique at B and $M R C$ scheme at $E R$.

3. For $M_{c}=M$, then $p_{\text {out }}$ is SOP corresponding to MRC sc heme at both $B$ and ER with single antenna Alice and $a P U$.

4. $P_{\text {out }_{v}}\left(\Psi_{t}\right)$ is the SOP as a function of threshold SNR corresponding to GSC scheme at legitimate receiver $B$ and $M R C$ technique at ER with a primary user. Similarly, $P_{\text {out }_{1}}\left(\Psi_{t}\right)$ is the SOP as a function of threshold SNR corresponding to SC technique at $B$ and MRC scheme at ER.

The average number of active branches $N_{b}$ with MSGSC is expressed as

where $n \operatorname{Pr}\left[N_{b}=n\right]$ is the PMF (probability mass function) of $N_{b}$ and given by

$\operatorname{Pr}\left[N_{b}=n\right]= \begin{cases}1-\operatorname{P}_{\Upsilon_{1}}\left(\Psi_{t}\right) & n=1 \\ \operatorname{P}_{\Upsilon_{n-1}}\left(\Psi_{t}\right)-P_{\Upsilon_{n}}\left(\Psi_{t}\right) & 2 \leq n \leq M_{c}-1 \\ \operatorname{P}_{\Upsilon_{M_{c}-1}}\left(\Psi_{t}\right) & n=M_{c}\end{cases}$

\subsection{Asymptotic secrecy outage probability}

Here, asymptotic nature of SOP in high SNR regime $\psi_{1} \rightarrow$ $\infty$ is considered. By applying ([31], Eq. 1.211.1), the firstorder expansion of $F_{\psi_{1}}^{\infty}$ is written as (27). Using (27) in (7), asymptotic SOP is calculated in (28). Here, we assume there are $N_{E}$ antennas at eavesdropper, i.e., $M=N_{E}$. 


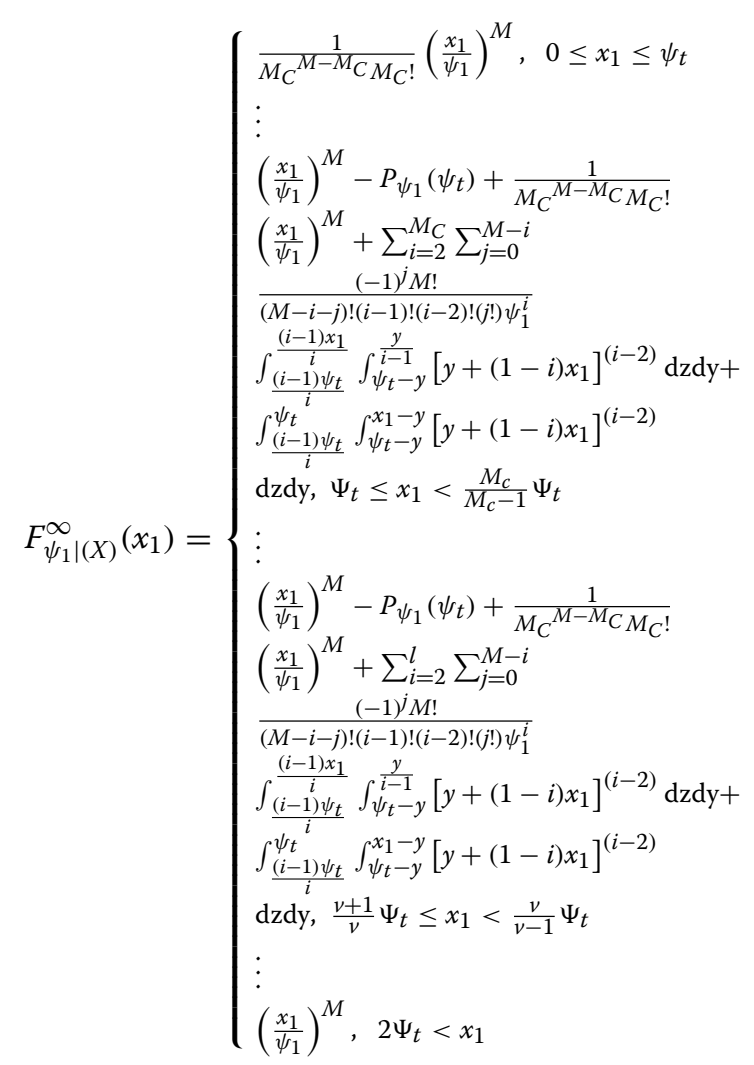

$P_{\psi_{1}}\left(\psi_{t}\right)=\sum_{i=0}^{M}\left(\begin{array}{c}M \\ i\end{array}\right) e^{\frac{-\psi_{t}}{\psi_{1}}}$

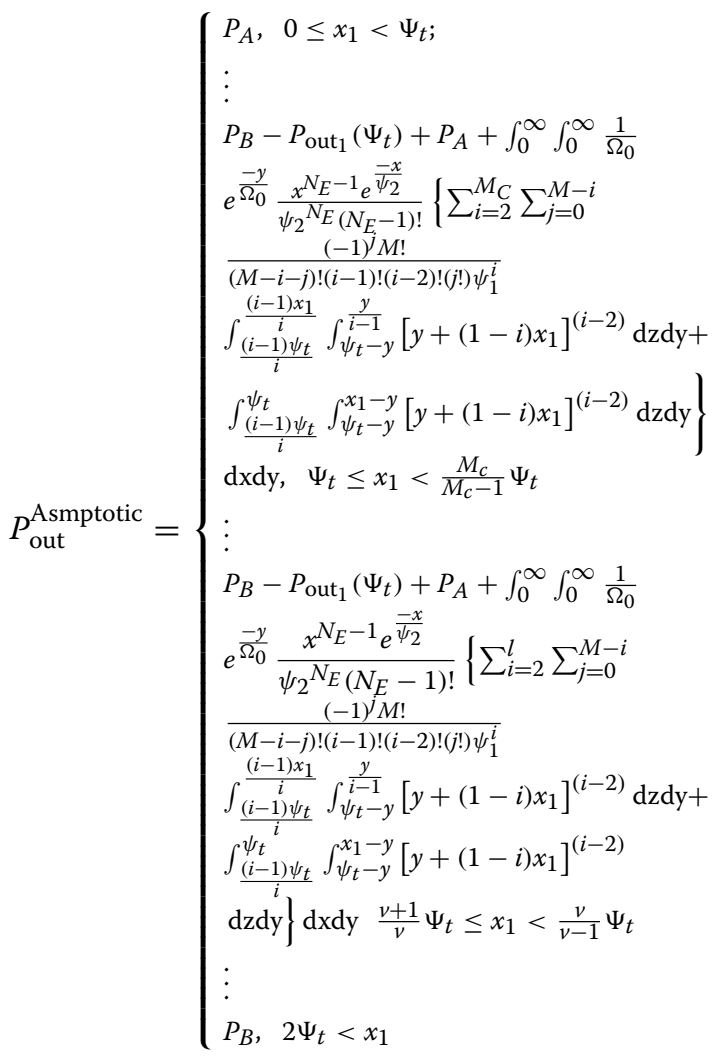

$$
P_{A}=\frac{1}{M_{C}{ }^{M-M_{C}} M_{C} !} P_{B}
$$

where

$$
\begin{array}{r}
\left(1-e^{\frac{-\sigma}{\Omega_{0}}}\right) \\
P_{B}=\frac{M}{\left(\psi_{2}\right)^{N_{E}\left(N_{E}-1\right)}} \sum_{k=0}^{M}\left(\begin{array}{c}
M \\
k
\end{array}\right)\left(\frac{2^{R s}-1}{\psi_{1}}\right)^{k}\left(\frac{2^{R s}}{\psi_{1}}\right)^{M-k} \\
\int_{0}^{\infty} x^{N_{E}-1+M-k} e^{\frac{-x}{\psi_{2}}} d x+\sum_{k=0}^{M}\left(\begin{array}{c}
M \\
k
\end{array}\right)\left(\frac{2^{R s}-1}{\sigma \psi_{1}}\right)^{k}\left(\frac{2^{R s}}{\sigma \psi_{1}}\right)^{M-k} \\
\int_{0}^{\infty} x^{N_{E}-1+M-k} e^{\frac{-x y}{\sigma \psi_{2}}} d x \frac{1}{\Omega_{0}} \int_{\sigma}^{\infty} e^{\frac{-y}{\Omega_{0}}} d y
\end{array}
$$

(28) can also be written as

$$
P_{\text {out }}^{\text {Asmptotic }}=\left(G_{A} \psi_{1}\right)^{-G_{D}}+O\left(\psi_{1}^{-G_{D}}\right)
$$

For $0 \leq x_{1} \leq \psi_{t}$, the secrecy diversity order is

$$
G_{D}=M \text {, }
$$

and the secrecy array gain is

$$
\begin{array}{r}
G_{A}=\left[\frac{1}{\left(M_{c}\right) ! M_{c}^{M-M_{c}}} \frac{\left(q+N_{E}-1\right) !}{\left(N_{E}-1\right) !} \sum_{q=0}^{M}\left(\begin{array}{c}
M \\
q
\end{array}\right)\left(2^{R s}-1\right)^{M-q}\right. \\
\left(2^{R s}\right)^{q} \gamma_{2} q\left(1-e^{-\frac{\sigma}{\Omega_{0}}}\right)+\frac{1}{\left(M_{c}\right) ! M_{c}^{M-M_{c}} \frac{\left(q+N_{E}-1\right) !}{\left(N_{E}-1\right) !}} \\
\sum_{q=0}^{M}\left(\begin{array}{c}
M \\
q
\end{array}\right)\left(2^{R s}-1\right)^{M-q}\left(2^{R s}\right)^{q}\left(\sigma \gamma_{2}\right)^{q} e^{\frac{-\sigma}{\Omega_{0}}} \sum_{t=0}^{M-1} \frac{(M-q)}{t !} \\
\left.\frac{(\sigma)^{t}}{\left(\frac{1}{\Omega_{0}}\right)^{M-q-t+1}}\right]^{\frac{-1}{M}}
\end{array}
$$

For $2 \Psi_{t}<x_{1}$, the secrecy diversity order is $G_{D}=M$ and secrecy array gain is

$$
\begin{array}{r}
G_{A 1}=\left[\frac{\left(q+N_{E}-1\right) !}{\left(N_{E}-1\right) !} \sum_{q=0}^{M}\left(\begin{array}{c}
M \\
q
\end{array}\right)\left(2^{R s}-1\right)^{M-q}\left(2^{R s}\right)^{q} \psi_{2}{ }^{q}\right. \\
\left(1-e^{-\frac{\sigma}{\Omega_{0}}}\right)+\frac{\left(q+N_{E}-1\right) !}{\left(N_{E}-1\right) !} \sum_{q=0}^{M}\left(\begin{array}{c}
M \\
q
\end{array}\right)\left(2^{R s}-1\right)^{M-q}\left(2^{R s}\right)^{q} \\
\left.\left(\sigma \psi_{2}\right)^{q} e^{\frac{-\sigma}{\Omega_{0}}} \sum_{t=0}^{M-1} \frac{(M-q)}{t !} \frac{(\psi)^{t}}{\left(\frac{1}{\Omega_{0}}\right)^{M-q-t+1}}\right]^{\frac{-1}{M}}
\end{array}
$$

According to (31), (32), (33), and (34), we have the following remarks to provide insight into the use of MS-GSC at secondary receiver.

Remark 2 As indicated in (28), asymptotic SOP approaches to zero as $\psi_{1} \rightarrow \infty$. Furthermore, one also can observe that all asymptotic curves tightly approximate the exact curves in high $\psi_{1}$ regime. 
Remark 3 The asymptotic result confirm that the secrecy diversity order is independent of $N_{E}$ and $\psi_{2}$, as indicated in (32). Note the secrecy outage probability increases with increasing $N_{E}$ and $\psi_{2}$. This confirms that the secrecy array gain in (33) and (34) is a decreasing function of $N_{E}$ and $\psi_{2}$.

Remark 4 The secrecy diversity order is independent of choice of $M_{c}$ i.e. the number of combined antennas in B. It is dependent on the number of available receiver antennas at the $B$.

\section{Numerical result}

Numerical results highlight the effect of number of diversity branches, average SNR of ER, and $\sigma$ on SOP. We assume $\Omega_{0}=1$ throughout this analysis. Exact and asymptotic curves are obtained from (15) and (28), respectively. These curves are also verified using Monte-Carlo simulations.

Figure 1 plots SOP of MS-GSC as given in (15) as a function of output threshold $R_{s}$ for different numbers of diversity branches $M_{c}$. Here, we assume $\Psi_{1}=30 \mathrm{~dB}$, $\Psi_{2}=10 \mathrm{~dB}, \Psi_{t}=7 \mathrm{~dB}, \sigma=1$, and $M=7$. We also plot SOP of 7/3-GSC for comparison. It is clear from the figure that SOP decreases as the number of diversity branches $M_{c}$ increases, especially when the output threshold $R_{s}$ is less than $\Psi_{t}$. When $R_{s}>\Psi_{t}$, the MS-GSC combiner try to increase the $\Psi_{\text {com }}$ above $\Psi_{t}$ and the SOP degrades. So, it is confirmed that the output threshold $R_{s}$ should be less than or equal to the threshold SNR $\Psi_{t}$. For $M_{c}=3$, SOP of MS-GSC is same as conventional GSC if $R_{s}>\Psi_{t}$.

Figure 2 plots SOP versus average SNR of the main channel $\Psi_{1}$ for different numbers of MS-GSC branches. As we see, SOP decreases as the number of MS-GSC branches $M_{c}$ increases.

Figure 3 plots the exact and asymptotic SOP obtained from (15) and (28) versus the average SNR of main channel $\Psi_{1}$ for different numbers of MS-GSC branches with variation in average SNR of ER's channel. From the figure, it is clear that asymptotic curves correlate with the exact result at high SNR regime. According to (32), the secrecy diversity order is independent of $N_{E}$ and only dependent on $M$. As $\Psi_{2}$ increases, secrecy performance degrades.

Figure 4 plots SOP for different values of $\sigma$. We see that SOP decreases with increasing $\sigma$. This is because of peak interference power constraint $\sigma=\frac{\Psi_{p}}{\Psi_{0}}=\frac{P_{I}}{P_{a v}}$, which leads to increase in average transmit power of Alice given by (8).

Figure 5 plots active MRC branches versus threshold SNR $\Psi_{t}$. We can see that active MRC branches increases as threshold SNR $\psi_{t}$ increases because it is very hard to increase the SNR of the combined $\Psi_{\text {com }}$ above $\Psi_{t}$. MSGSC requires less active MRC branches as compared to GSC that result in less processing power. The percentage

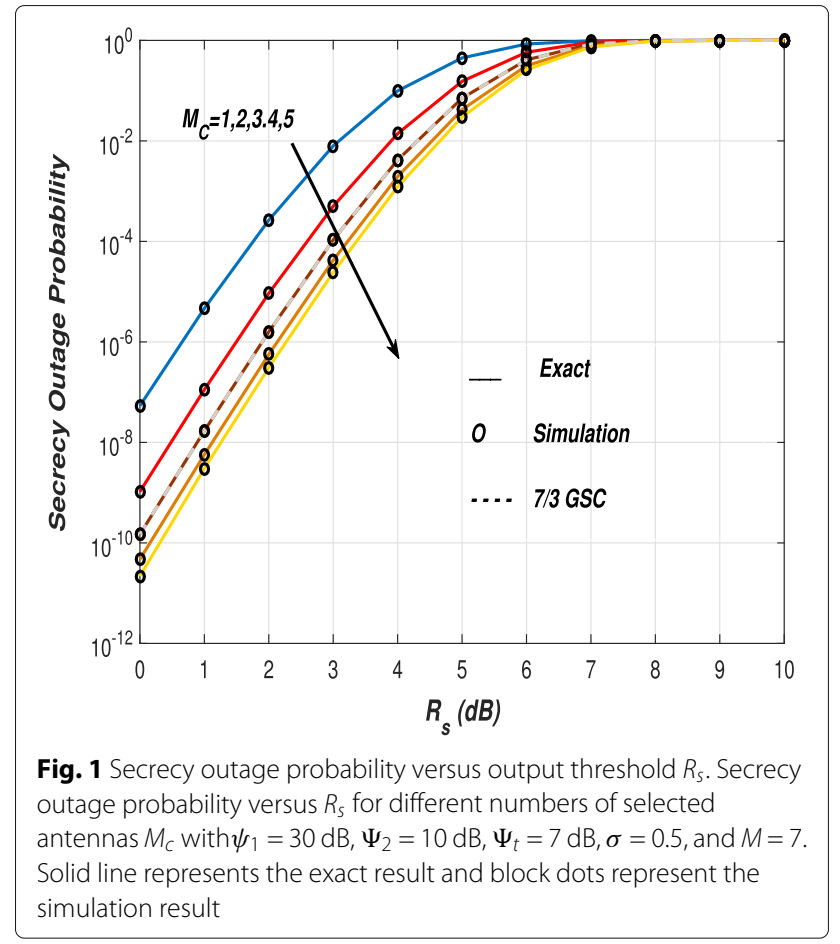

of power saving for different number of antenna is shown in Table 1.

\section{Conclusions}

We proposed MS-GSC/MRC protocol for underlay cognitive radio network. We consider SOP as a main parameter to analyze secrecy of proposed system. We derived closedform expression for exact and asymptotic SOP. From

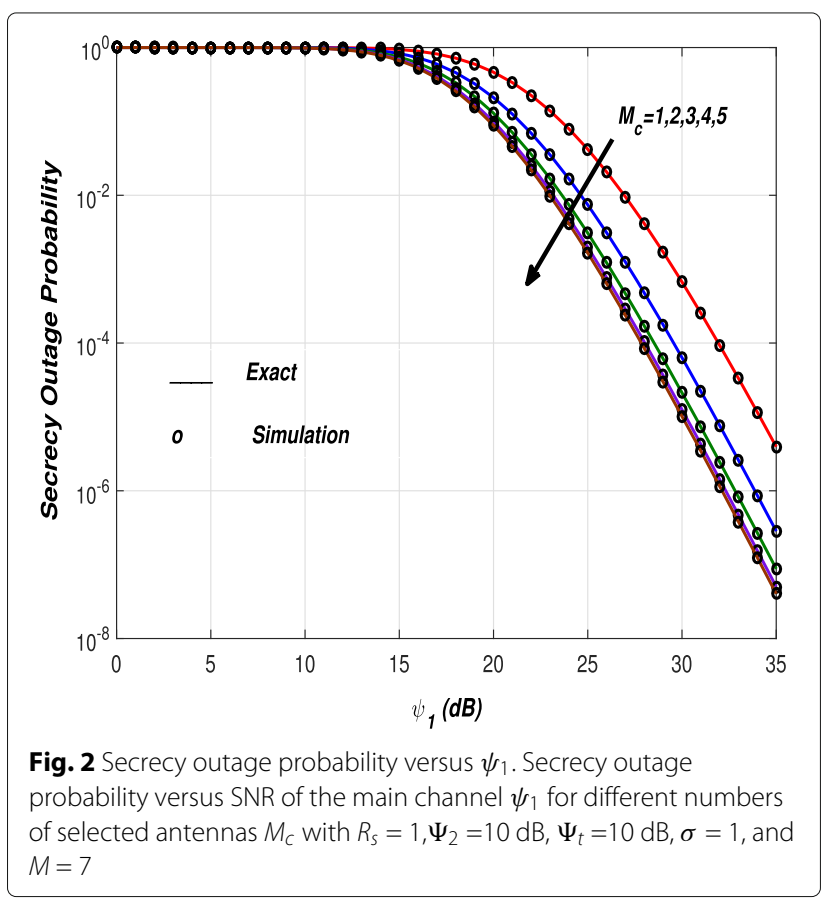




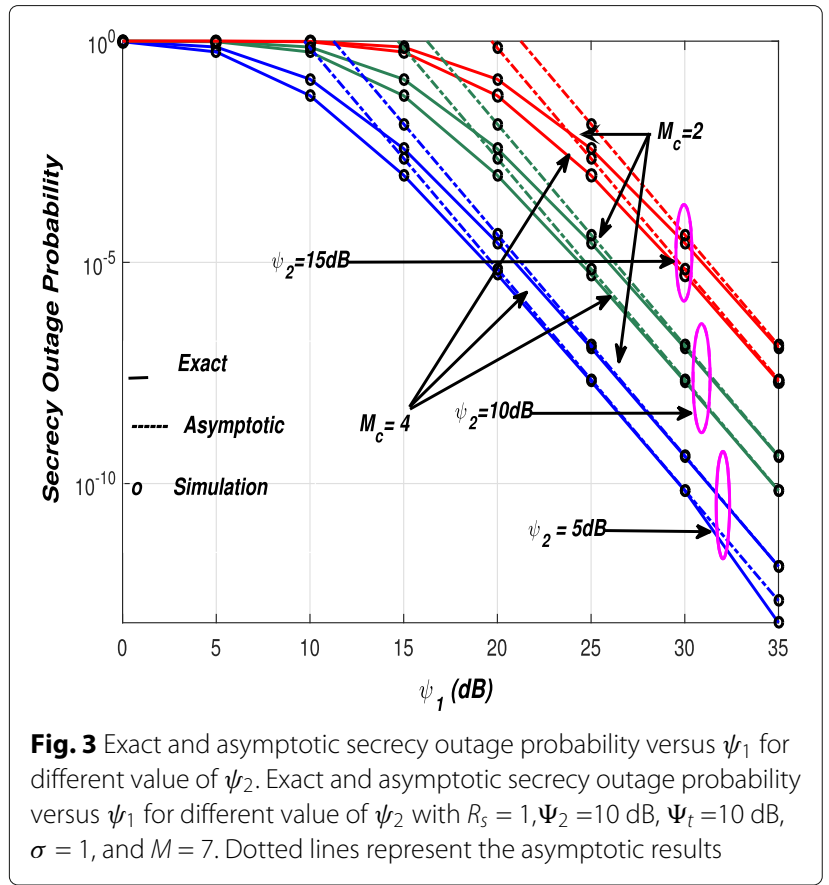

numerical results, it has been concluded that MS-GSC saves more processing power in comparison to conventional GSC, and the number of selected antennas $M_{c}$ and $\sigma$ has positive impact on SOP.

\section{Appendix}

Based on (9), $Y \leq \frac{\Psi_{p}}{\Psi_{0}}, \Psi_{M}=\Psi_{0} X_{M}, \Psi_{E}=\Psi_{0} X_{E}$ and when $Y>\frac{\Psi_{p}}{\Psi_{0}}, \Psi_{M}=\frac{\Psi_{p}}{Y} X_{M}, \Psi_{E}=\frac{\Psi_{p}}{Y} X_{E}$. Hence, SOP for proposed system can be calculated as

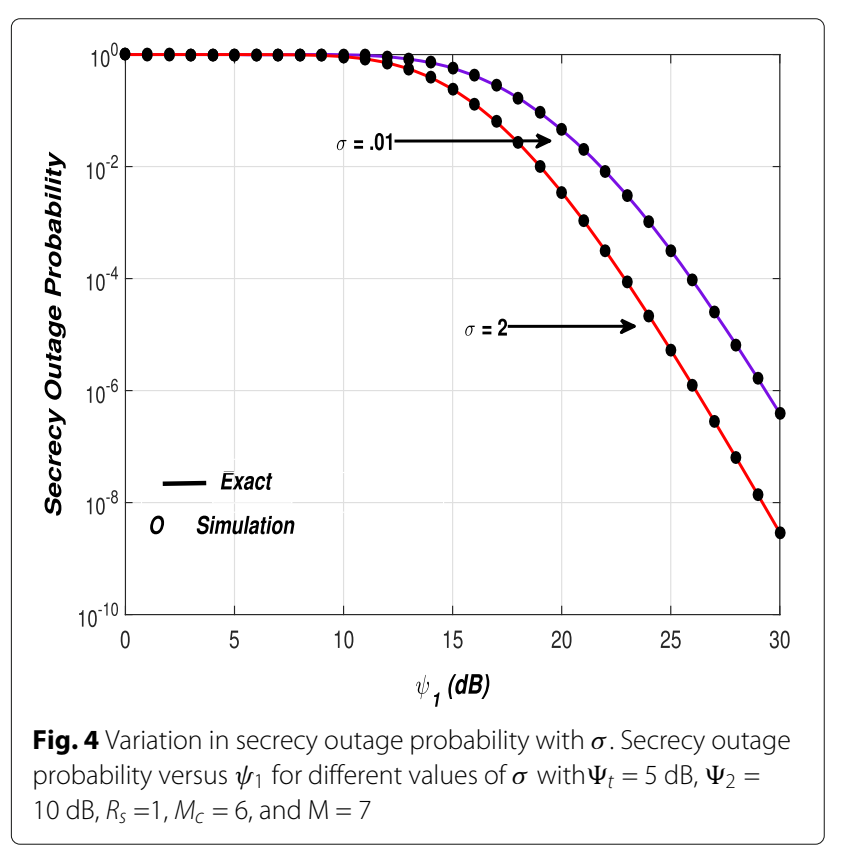

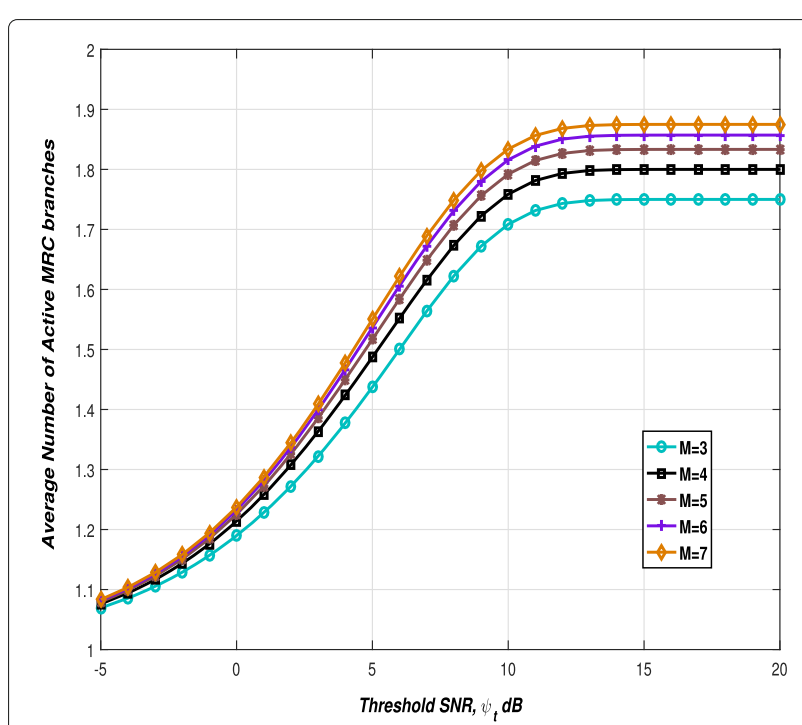

Fig. 5 Average number of active MRC branches. Active MRC branches versus output SNR for different numbers of antennas $M$ with $M_{C}=2$ and $\Psi_{1}=10 \mathrm{~dB}$

$$
\begin{aligned}
& P_{\text {out }}=\underbrace{\int_{0}^{\sigma} \int_{0}^{\infty} F_{\psi_{1}\left(X_{1}=x_{1}\right)}\left(x_{1}\right) f_{\psi_{2}(X=x)}(x) f_{Y}(y) \mathrm{dxdy}}_{J_{1}} \\
& \underbrace{\int_{\sigma}^{\infty} \int_{0}^{\infty} F_{\psi_{1}\left(X_{1}=x_{1}\right)}\left(x_{1}\right) f_{\psi_{2}(X=x)}(x) f_{Y}(y) \mathrm{dxdy}}_{J_{2}}
\end{aligned}
$$

for $X_{1} \leq \frac{\psi_{p}}{\psi_{0}}$, substituting Eqs. (10), (12), and (14) in Eq. (36), $J_{1}$ can be calculated as

$$
J_{1}= \begin{cases}P_{\text {out }_{A 1}} & 0 \leq x_{1}<\Psi_{t} \\ P_{\text {out }_{B 1}} & \Psi_{t} \leq x_{1}<\frac{M_{c}}{M_{c}-1} \Psi_{t} \\ \vdots \\ P_{\text {out }_{C 1}} & \frac{v+1}{v} \Psi_{t} \leq x_{1}<\frac{v}{v-1} \Psi_{t} \\ \vdots \\ P_{\text {out }_{D 1}} 2 \Psi_{t}<x_{1}\end{cases}
$$

Table 1 Power saving by MS-GSC

\begin{tabular}{lllll}
\hline Case & Number of antenna & $M_{c}$ & Active MRC branches & Power saving (\%) \\
\hline 1 & 3 & 2 & 1.750 & 12.5 \\
2 & 4 & 2 & 1.800 & 10 \\
3 & 5 & 2 & 1.833 & 8.35 \\
4 & 6 & 2 & 1.857 & 7.15 \\
5 & 7 & 2 & 1.875 & 6.25 \\
\hline
\end{tabular}




$$
\begin{aligned}
& P_{\text {out }_{A 1}}=\frac{M !}{M_{C} !\left(M-M_{C}\right) !}\left\{( 1 - e ^ { \frac { \sigma } { S _ { 0 } } ) } ) \left(1-\sum_{k=0}^{L_{c}-1} \sum_{n=0}^{k} \mu_{1} e^{\left(\frac{-\left(2^{R_{S}}-1\right)}{\psi_{1}}\right)}\right.\right. \\
& +\sum_{v=1}^{M-M_{c}} C_{2} C_{3}-\sum_{v=1}^{M-M_{c}} \sum_{m=0}^{M_{c}-2} C_{2} C_{4} \\
& -\sum_{v=1}^{M-M_{c}} C_{2} C_{4} \beta_{1} e^{-\left(\frac{\left(2^{R s}-1\right)\left(1+\frac{V}{M_{c}}\right)}{w_{1}}\right)} \\
& \left.\left.+\sum_{v=1}^{M-M_{c}} \sum_{m=0}^{M_{c}-2} \sum_{k=0}^{m} \sum_{n=0}^{k} C_{2} C_{4} \mu_{1} e^{-\left(\frac{2 R S-1}{\psi_{1}}\right)}\right)\right\}
\end{aligned}
$$$$
P_{\text {out }_{B 1}}=P_{\text {out }_{D 1}}-P_{\text {out }_{1 A}}\left(\Psi_{t}\right)+P_{\text {out }_{M c}}\left(\Psi_{t}\right)+\sum_{w=2}^{M_{c}} P_{\text {out }_{M 1}}
$$

$$
P_{\text {out }_{M}}=I_{1}
$$

$$
\begin{gathered}
P_{\operatorname{out}_{1 A}}\left(\Psi_{t}\right)=\sum_{v=0}^{M}\left(\begin{array}{c}
M \\
v
\end{array}\right)(-1)^{v} e^{\frac{-v \psi_{t}}{\psi_{1}}}\left(1-e^{\frac{-\sigma}{\Omega_{0}}}\right) \\
P_{\operatorname{out}_{M_{c 1}}}\left(\Psi_{t}\right)=\left(1-e^{\frac{-\sigma}{\Omega_{0}}}\right) \frac{M !}{\left(M-M_{c}\right) ! M_{c} !}\left\{1-e^{\frac{-\Psi_{t}}{\Psi_{1}}} \sum_{k=0}^{M_{c}-1} \frac{1}{k !}\right. \\
\left(\frac{\Psi_{t}}{\Psi_{1}}\right)^{k}+\sum_{v=1}^{M-M_{c}} C_{2}\left[C_{3}\left(1-e^{-\left(1+\frac{v}{M_{c}}\right) \frac{\Psi_{t}}{\Psi_{1}}}\right)\right. \\
\left.\left.-\sum_{m=0}^{M_{c}-2} C_{4}\left(1-e^{\frac{-\Psi_{t}}{\Psi_{1}}} \sum_{k=0}^{m} \frac{1}{k !}\left(\frac{\Psi_{t}}{\Psi_{1}}\right)^{k}\right)\right]\right\}
\end{gathered}
$$

$$
P_{\text {out }_{C 1}}=P_{\text {out }_{D}}-P_{\text {out }_{1 A}}\left(\Psi_{t}\right)+P_{\text {out }_{v 1}}\left(\Psi_{t}\right)+\sum_{w=2}^{v} P_{\text {out }_{M 1}}
$$

$$
P_{\text {out }_{D 1}}=\left(1-e^{\frac{-\sigma}{\Omega_{0}}}\right) \sum_{v=0}^{M}\left(\begin{array}{c}
M \\
v
\end{array}\right)(-1)^{v} \frac{e^{-\left(\frac{-v\left(2^{R s}-1\right)}{\Psi_{1}}\right)}}{\left(\Psi_{2}\right)^{M}\left(\frac{v 2^{R s}}{\Psi_{1}}+\frac{1}{\Psi_{2}}\right)^{M}}
$$$$
P_{\text {out }_{v 1}}\left(\Psi_{t}\right)=\left(1-e^{\frac{-\sigma}{\Omega_{0}}}\right) \frac{M !}{(M-v) ! v !}\left\{1-e^{\frac{-\Psi_{t}}{\Psi_{1}}} \sum_{k=0}^{v-1} \frac{1}{k !}\left(\frac{\Psi_{t}}{\Psi_{1}}\right)^{k}\right.
$$$$
+\sum_{v=1}^{M-v}(-1)^{2 v-1} \frac{(M-v) !}{(M-v-v) ! v !} \times\left[\frac{1}{2}\left[1-e^{-\frac{2 \Psi_{t}}{\Psi_{1}}}\right]\right.
$$$$
\left.\left.-\sum_{m=0}^{\nu-2}(-1)^{m}\left(1-e^{\frac{-\Psi_{t}}{\Psi_{1}}} \sum_{k=0}^{m} \frac{1}{k !}\left(\frac{\Psi_{t}}{\Psi_{1}}\right)^{k}\right)\right]\right\}
$$

$$
\begin{array}{r}
I_{1}=\left(1-e^{\frac{-\sigma}{\Omega_{0}}}\right)\left(r _ { 1 } \left[r_{5}-\frac{e^{\frac{2^{R S}-1}{\Psi_{1}}}}{(M-1) !} \Psi_{2}^{M} \sum_{a=0}^{m-v}\left(2^{R s}-1\right)^{m-v-a}\left(2^{R s}\right)^{a} \frac{(a+M-1) !}{\left(\frac{2^{R s}}{\Psi_{1}}+\frac{1}{\Psi_{2}}\right)^{a+M}+\frac{e^{\frac{2^{R S}-1}{\gamma_{1}}}}{(M-1) ! \Psi_{2}^{M}} \sum_{a=0}^{m-l}\left(2^{R s}-1\right)^{m-v-a}\left(2^{R s}\right)^{a}}\right.\right. \\
\left.\sum_{b=0}^{w-1-k+v}\left(\begin{array}{c}
w-1-k+v \\
b
\end{array}\right)\left(\frac{(w-1)\left(2^{R s}-1\right)}{w \Psi_{t}}\right)^{(w-1-k+v-b)}\left(\frac{(w-1)\left(2^{R s}\right)}{w \Psi_{t}}\right)^{b} \frac{(a+b+M-1) !}{\left(2^{R s}+\frac{1}{\Psi_{1}}\right)^{a+b+M}}\right]+r_{2}\left[r_{3}-\sum_{a=0}^{m-v}\right.
\end{array}
$$

$$
\begin{aligned}
& \sum_{n=0}^{w-2-k+v} \sum_{b=0}^{n}\left(\begin{array}{c}
m-v \\
a
\end{array}\right)\left(2^{R s}-1\right)^{m-v-a}\left(2^{R s}\right)^{a} e^{\left(\frac{(w+j)\left(2^{R s}-1\right)}{w \Psi_{1}}\right)}\left(\begin{array}{l}
n \\
a
\end{array}\right) \frac{1}{n !}\left(\frac{-(w-1) j\left(2^{R s}-1\right)}{w \Psi_{1}}\right)^{n-b}\left(\frac{-(w-1) j\left(2^{R s}\right)}{w \Psi_{1}}\right)^{n} \frac{(a+b+M-1) !}{(M-1) ! \Psi_{2} M}
\end{aligned}
$$

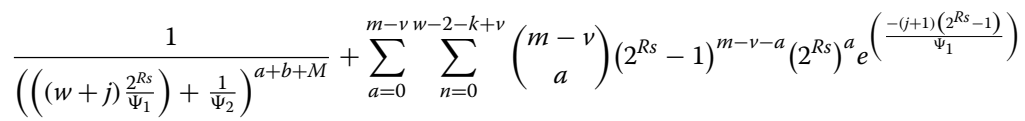$$
\left.e^{-j \frac{\Psi_{t}}{\Psi_{1}}} r_{25} \frac{(a+M-1) !}{(M-1) ! \Psi_{2}^{M}} \frac{1}{\left(\left((w+j) \frac{2^{R s}}{\Psi_{1}}\right)+\frac{1}{\Psi_{2}}\right)^{a+M}}\right]+r_{4}\left[r_{10}-\sum_{n=0}^{w-2-k+m} \sum_{b=0}^{n} e^{\left(\frac{-(w+j)\left(2^{R s}-1\right)}{w \psi_{1}}\right)} e^{\left(\frac{-(w+j)\left(2^{R s}\right)}{w \Psi_{1}}\right)} \frac{1}{n !}\left(\frac{(w+j)}{w \Psi_{1}}\right)^{n}\left(\begin{array}{l}
n \\
b
\end{array}\right)\left(2^{R s}-1\right)^{n-b}\right.
$$

$$
\left.\left.\left(2^{R s}\right)^{b} \frac{(a+M-1) !}{(M-1) ! \Psi_{2}^{M}} \frac{1}{\left(\frac{(w+j) 2^{R s}}{w \Psi_{1}}+\frac{1}{\Psi_{2}}\right)^{b+M}}\right]\right)
$$

$$
\begin{array}{r}
r_{1}=\frac{M !}{(M-w) !(w-1) !} \sum_{k=0}^{w-2} \frac{(1-w)^{k}}{(w-2-k) !} \sum_{m=0}^{k} \frac{1}{\Psi_{1}^{w-1-k+m}} \sum_{v=0}^{m} \frac{(-1)^{v}}{(m-v) ! l !} \frac{\Psi_{t}^{w-1-k+v}}{w-1-k+v}, \quad r_{5}=e^{\frac{-\Psi_{t}}{\Psi_{1}}} \Psi_{t}^{m-v}\left(1-\left(\frac{w-1}{w}\right)^{w-1-k+v}\right) \\
r_{2}=\sum_{j=1}^{M-w} \frac{(-1)^{j} M !}{(L-w-j) !(w-1) ! j ! \Psi_{1}^{w}} \sum_{k=0}^{w-2} \frac{(1-w)^{k}}{(w-2-k) !} \sum_{m=0}^{k}\left(\frac{j+1}{\gamma_{1}}\right)^{m-k-1} \sum_{v=0}^{m} \frac{(-1)^{l}(w-2-k+l) !}{(m-v) ! v !}\left(\frac{-\Psi_{t}}{j}\right)^{w-1-k+v} \\
r_{3}=\Psi_{t}^{m-v}\left(e^{\frac{-(w+j) \Psi_{t}}{w}} \sum_{n=0}^{i-2-k+l} \frac{1}{n !}\left(\frac{-(w-1) j \Psi_{t}}{w \Psi_{1}}\right)^{n}-e^{\frac{-\Psi_{t}}{\Psi_{1}}} \sum_{n=0}^{w-2-k+l} \frac{1}{n !}\left(\frac{-j \Psi_{t}}{\Psi_{1}}\right)^{n}\right) \\
r_{4}=\sum_{j=0}^{M-w} \frac{(-1)^{j} M !}{(M-w-j) !(w-1) ! j ! \Psi_{1}^{w}} \sum_{k=0}^{w-2} \frac{(1-w)^{k}}{(w-2-k) !} \sum_{m=0}^{k} \frac{(w-2-k+m) !}{m !(w-1)^{m}}\left(\frac{j+1}{\Psi_{1}}\right)^{m-k-1}\left(\frac{(w-1) \Psi_{1}}{w+j}\right)^{w-1-k+m} \\
r_{10}=e^{\frac{-\left(w+j \Psi_{t}\right.}{w \psi_{1}}} \sum_{n=0}^{w-2-k+m} \frac{1}{n !}\left(\frac{(w+j) \Psi_{t}}{w \Psi_{1}}\right)^{n}, \quad r_{25}=\frac{1}{n !}\left(\frac{-j \Psi_{t}}{\Psi_{1}}\right)^{n}
\end{array}
$$


$I_{1}$ given in (20) can be calculated using ([22], Eq. (30)).

$J_{2}=\int_{\frac{\psi_{p}}{\gamma_{0}}}^{\infty} \int_{0}^{\infty} F_{\gamma_{1}\left(X_{1}=x_{1}\right)}\left(x_{1}\right) f_{\gamma_{2}(X=x)}(x) f_{Y}(y) \mathrm{dxdy}$

for $X_{1}>\frac{\gamma_{p}}{\gamma_{0}}$

By Eqs. (10), (12), and (14) in Eq. (36) and using $\int_{\rho}^{\infty} x^{m} e^{-\mu x} d x=e^{-\rho \mu} \sum_{p=0}^{m} \frac{m !}{p !} \frac{\rho^{k}}{\mu^{m-p+1}}, J_{2}$ can be given as

$$
\begin{aligned}
& J_{2}= \begin{cases}P_{\text {out }_{A 2}} & 0 \leq x_{1}<\Psi_{t} \\
P_{\text {out }_{B 2}} & \Psi_{t} \leq x_{1}<\frac{M_{c}}{M_{c}-1} \Psi_{t} \\
\vdots & \\
P_{\text {out }_{C 2}} & \frac{v+1}{v} \Psi_{t} \leq x_{1}<\frac{v}{v-1} \Psi_{t} \\
\vdots \\
P_{\text {out }_{D 2}} 2 \Psi_{t}<x_{1}\end{cases} \\
& p_{\text {out }_{A 2}}=\frac{M !}{M_{C} !\left(M-M_{C}\right) !}\left\{e^{\frac{-\sigma}{\Omega_{0}}}-\sum_{k=0}^{M_{c}-1} \sum_{n=0}^{k} \sum_{p=0}^{k-n} Q_{1} Q_{2}\right. \\
& e^{-\sigma\left(\frac{2^{R s}-1}{\sigma \Psi_{1}}+\frac{1}{\Omega_{0}}\right)}+\sum_{\nu=1}^{M-M_{c}} C_{2} C_{3} e^{-\left(\frac{\sigma}{\Omega_{0}}\right)}-\sum_{\nu=1}^{M-M_{c}} C_{2} C_{3} \beta_{2} \\
& e^{-\sigma\left(\left(1+\frac{M}{M_{c}}\right)\left(\frac{2^{R s}-1}{\sigma \psi_{1}}\right)+\frac{1}{\Omega_{0}}\right)}-\sum_{\nu=1}^{M-M_{c} M_{c}-2} \sum_{m=0} C_{2} C_{4} e^{-\left(\frac{\sigma}{\Omega_{0}}\right)} \\
& \left.+\sum_{v=1}^{M-M_{c}} \sum_{m=0}^{M_{c}-2} \sum_{k=0}^{m} \sum_{n=0}^{k} \sum_{p=0}^{k-n} C_{2} C_{4} Q_{1} Q_{2} e^{-\sigma\left(\frac{2^{R s}-1}{\sigma \psi_{1}}+\frac{1}{\Omega_{0}}\right)}\right\} \\
& P_{\text {out }_{B 2}}=P_{\text {out }_{D 2}}-P_{\text {out }_{2 A}}\left(\Psi_{t}\right)+P_{\text {out }_{M_{c 2}}}\left(\Psi_{t}\right)+\sum_{w=2}^{M_{c}} P_{\text {out }_{M}} \\
& P_{\text {out }_{D 2}}=\sum_{v=0}^{M}\left(\begin{array}{c}
M \\
v
\end{array}\right)(-1)^{v}
\end{aligned}
$$

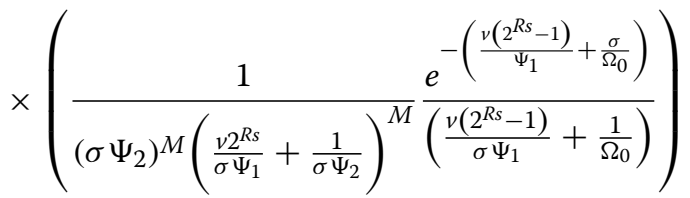

$$
\begin{gathered}
P_{\text {out }_{M_{c 2}}\left(\Psi_{t}\right)}=\frac{M !}{\left(M-M_{c}\right) ! M_{c} !}\left[e ^ { \frac { - \sigma } { \Omega _ { 0 } } } \left(1+\sum_{v=1}^{M-M_{c}} C_{2} C_{3}\right.\right. \\
\left.-\sum_{v=1}^{M-M_{c}} \sum_{m=0}^{M_{c}-2} C_{2} C_{4}\right)-\frac{1}{\Omega_{0}} \sum_{k=0}^{M_{c}-1} \sum_{l=0}^{k} \frac{1}{k !}\left(\frac{\psi_{t}}{\sigma \psi_{1}}\right)^{k} \\
e^{-\left(\frac{\psi_{t}}{\psi_{1}}+\frac{\sigma}{\Omega_{0}}\right)} \frac{k !}{l !} \frac{(\sigma)^{l}}{\left(\frac{\psi_{t}}{\sigma \psi_{1}}+\frac{1}{\Omega_{0}}\right)}\left(1-\sum_{v=1}^{k-l+1} \sum_{m=0}^{M-M_{c} M_{c}-2} C_{2} C_{4}\right) \\
\left.-\frac{1}{\Omega_{0}} \sum_{v=1}^{M-M_{c}} C_{2} C_{3} \frac{e^{-\left(\frac{\psi_{t}}{C_{3} \sigma \psi_{1}}+\frac{\sigma}{\Omega_{0}}\right)}}{\frac{\psi_{t}}{C_{3} \sigma \psi_{1}}+\frac{\sigma}{\Omega_{0}}}\right]
\end{gathered}
$$$$
P_{\text {out }_{C 2}}=P_{\text {out }_{D 2}}-P_{\text {out }_{1}}\left(\Psi_{t}\right)+P_{\text {out }_{v 2}}\left(\Psi_{t}\right)+\sum_{w=2}^{v} P_{\text {out }_{M 1}}
$$

$$
\begin{aligned}
& P_{\text {out }_{v 2}}\left(\Psi_{t}\right)=\frac{M !}{(M-v) ! v}\left[e ^ { \frac { - \sigma } { \Omega _ { 0 } } } \left(1+\sum_{v=1}^{M-v} \frac{1}{2} K_{1}-\sum_{v=1}^{M-v}\right.\right. \\
& \left.\sum_{m=0}^{v-2} K_{1}(-1)^{m}\right)-\frac{1}{\Omega_{0}} \sum_{k=0}^{\nu-1} \sum_{l=0}^{k} \frac{1}{k !}\left(\frac{\psi_{t}}{\sigma \psi_{1}}\right)^{k} e^{-\left(\frac{\psi_{t}}{\psi_{1}}+\frac{\sigma}{\Omega_{0}}\right)} \frac{k !}{l !} \\
& \frac{(\sigma)^{l}}{\left[\frac{\psi_{t}}{\psi_{1} \sigma}+\frac{1}{\Omega_{0}}\right]^{k-l+1}}\left(1-\sum_{v=1}^{M-v} \sum_{m=0}^{v-2} K_{1}(-1)^{m}\right) \\
& \left.-\frac{1}{\Omega_{0}} \sum_{\nu=1}^{M-v} \frac{1}{2} K_{1} \frac{e^{-\left(\frac{2 \psi_{t}}{\psi_{1}}+\frac{\sigma}{\Omega_{0}}\right)}}{\left(\frac{2 \psi_{t}}{\sigma \psi_{1}}+\frac{1}{\Omega_{0}}\right)}\right]
\end{aligned}
$$

$$
P_{\text {out }_{M 2}}=I_{2}
$$




$$
\begin{aligned}
& I_{2}=\left(\left[r_{1}^{\prime} r_{5}^{\prime} \sum_{c=0}^{w-1-k+m} \frac{(w-1-k+m) !}{c !} \frac{(\sigma)^{c} e^{\left(-\sigma \frac{\Psi_{t}}{\sigma \Psi_{1}}+\frac{1}{\Omega_{0}}\right)}}{\left(\frac{\Psi_{t}}{\sigma \Psi_{1}}+\frac{1}{\Omega_{0}}\right)^{w-k+m-c}}-\frac{r_{1}^{\prime}}{(M-1) !\left(\sigma \Psi_{1}\right)^{M}} \sum_{a=0}^{m-v}\left(2^{R s}-1\right)^{m-v-a}\left(2^{R s}\right)^{a} \frac{(a+M-1) !}{\left(\frac{2^{R s}}{\sigma \Psi_{1}}+\frac{1}{\sigma \gamma_{2}}\right)^{a+M}}\left(\begin{array}{c}
m-v \\
a
\end{array}\right)\right.\right. \\
& e^{-\sigma\left(\frac{\left(2^{R s}-1\right)}{\sigma \Psi_{1}}+\frac{1}{\Omega_{0}}\right)} \sum_{d=0}^{w-1-k+m+a} \frac{(w-1-k+m+a) !}{d !} \frac{(\sigma)^{d}}{\left(\frac{2^{R s}-1}{\sigma \Psi_{1}}+\frac{1}{\Omega_{0}}\right)^{w-k+m+a}}+\frac{r_{1}^{\prime}}{(M-1) !\left(\sigma \Psi_{1}\right)^{M}} \sum_{a=0}^{m-v}\left(\begin{array}{c}
m-v \\
a
\end{array}\right) \\
& \left(2^{R s}-1\right)^{m-v-a}\left(2^{R s}\right)^{a} \sum_{b=0}^{w-1-k+l}\left(\begin{array}{c}
w-1-k+l \\
a
\end{array}\right)\left(\frac{(w-1)\left(2^{R s}-1\right)}{s \Psi_{t}}\right)^{(w-1-k+v-b)}\left(\frac{(v-1)\left(2^{R s}\right)}{w \gamma_{t}}\right)^{b} \frac{(a+b+M-1) !}{\left(\frac{2^{R s}}{\sigma \Psi_{1}}+\frac{1}{\sigma \Psi_{2}}\right)^{a+b+M}} \\
& \left.e^{-\sigma\left(\frac{\left(2^{R s}-1\right)}{\sigma \Psi_{1}}+\frac{1}{\Omega_{0}}\right)} \sum_{e=0}^{w-1-k+m-a-b} \frac{(w-1-k+m-a-b) !}{e !} \frac{(\sigma)^{e}}{\left(\frac{2^{R s}-1}{\sigma \Psi_{1}}+\frac{1}{\Omega_{0}}\right)^{w-k+m-a-b-e}}\right] \\
& +\left[r_{2}^{\prime} \Psi_{t}{ }^{m-v} \sum_{n=0}^{w-2-k+v} \frac{1}{n !}\left(\frac{-(w-1) j \Psi_{t}}{\sigma \Psi_{1} \Psi_{t}}\right)^{n} e^{-\sigma\left(\frac{(w+j) \Psi_{t}}{w \sigma \Psi_{t}}+\frac{1}{\Omega_{0}}\right)} \sum_{f=0}^{n+m-v} \frac{(n+m-v) !}{f !}\right. \\
& \frac{(\sigma)^{f}}{\left(\frac{(w+j) \Psi_{t}}{w \sigma \Psi_{t}}+\frac{1}{\Omega_{0}}\right)}-r_{2}^{\prime} \Psi_{t}{ }^{m-v} \sum_{n=0}^{w-2-k+l} \frac{1}{n !}\left(\frac{-j \Psi_{t}}{\sigma \Psi_{1}}\right)^{n} \sum_{g=0}^{n+m-v} \frac{(n+m-v) !}{g !} \frac{(\sigma)^{g}}{\left(\frac{\Psi_{t}}{\sigma \Psi_{1}}+\left(\frac{1}{\Omega_{0}}\right)\right)^{n+m-v-g+1}} \\
& -r_{2}^{\prime} \sum_{a=0}^{m-v} \sum_{n=0}^{w-2-k+v} \sum_{b=0}^{n}\left(\begin{array}{c}
m-v \\
a
\end{array}\right)\left(2^{R s}-1\right)^{m-v-a}\left(2^{R s}\right)^{a}\left(\begin{array}{l}
n \\
b
\end{array}\right) \frac{1}{n !}\left(\frac{-j(w-1)\left(2^{R s}-1\right)}{w \sigma \Psi_{1}}\right)^{n-b}\left(\frac{-j(w-1)\left(2^{R s}\right)}{w \sigma \Psi_{1}}\right)^{b} \\
& \frac{(a+b+M-1) !}{(M-1) !\left(\sigma \Psi_{2}\right)^{M}} \frac{1}{\left(\frac{(w+j) 2^{R s}}{\sigma \Psi_{1}}+\frac{1}{\sigma \Psi_{2}}\right)^{a+b+M}} e^{-\sigma\left(\frac{(w+j)\left(2^{R s}-1\right)}{w \Psi_{t}}+\frac{1}{\Omega_{0}}\right)} \sum_{h=0}^{n-a-b} \frac{(n-a-b) !}{h !} \frac{(\sigma)^{h}}{\left(\frac{(w+j)\left(2^{R s}-1\right)}{w \Psi_{t}}+\frac{1}{\Omega_{0}}\right)^{n-a-b+h+1}}+\sum_{a=0}^{m-v} \\
& \sum_{n=0}^{w-2-k+v} r_{2}^{\prime}\left(\begin{array}{c}
m-v \\
a
\end{array}\right)\left(2^{R s}-1\right)^{m-v-a}\left(2^{R s}\right)^{a} r_{25}^{\prime} \frac{(a+M-1) !}{(M-1) !\left(\Psi_{2} \sigma\right)^{M}} \frac{1}{\left(\frac{(j+1)\left(2^{R s}\right)}{\sigma \Psi_{1}}+\frac{1}{\sigma \Psi_{2}}\right)^{a+M}} e^{-\sigma\left(\frac{(j+1)\left(2^{R s}-1\right)}{\sigma \Psi_{1}}+\frac{j \Psi_{t}}{\sigma \psi_{1}}+\frac{1}{\Omega_{0}}\right)^{m-v+n-a}} \sum_{q=0}^{\frac{(m-v+n-a) !}{q !}} \\
& \left.\frac{(\sigma)^{p}}{\left(\frac{(j+1)\left(2^{R s}-1\right)}{\sigma \Psi_{1}}+\frac{j \Psi_{t}}{\sigma \Psi_{1}}+\frac{1}{\Omega_{0}}\right)^{m-v+n-a-q+1}}\right]+\left[\frac{r_{4}^{\prime}}{(M-1) !\left(\sigma \Psi_{2}\right)^{M}} \sum_{n=0}^{w-2-k+m} \frac{1}{n !}\left(\frac{(w+j) \Psi_{t}}{w \sigma \Psi_{1}}\right)^{n} e^{-\sigma\left(\frac{(w+j) \Psi_{t}}{w \sigma \Psi_{1}}+\frac{1}{\Omega_{0}}\right)}\right. \\
& \sum_{r=0}^{n} \frac{n !}{r !} \frac{(\sigma)^{r}}{\left(\frac{(w+j) \Psi_{t}}{w \sigma \Psi_{1}}+\frac{1}{\Omega_{0}}\right)^{n-r+1}}-\frac{r_{4}^{\prime}}{(M-1) !\left(\sigma \Psi_{2}\right)^{M}} \sum_{n=0}^{w-2-k+m} \sum_{b=0}^{n} \frac{1}{n !}\left(\frac{(w+j)}{w \sigma \Psi_{1}}\right)^{n}\left(\begin{array}{l}
n \\
b
\end{array}\right) \\
& \left.\left.\left(2^{R s}-1\right)^{n-b}\left(2^{R s}\right)^{b} \frac{(b+M-1) !}{\left(\frac{(w+j) 2^{R s}}{w \sigma \Psi_{1}}+\frac{1}{\sigma \Psi_{2}}\right)^{b+M}} e^{-\sigma\left(\frac{(w+j)\left(2^{R s}-1\right)}{w \sigma \Psi_{1}}+\frac{(w+j) 2^{R s}}{w \sigma \Psi_{1}}+\frac{1}{\Omega_{0}}\right)} \sum_{s=0}^{n-b-M} \frac{(n-b-M) !}{s !} \frac{(\sigma)^{s}}{\left(\frac{(w+j)\left(2^{R s}-1\right)}{i \sigma \Psi_{1}}+\frac{(w+j) 2^{R s}}{w \sigma \Psi_{1}}+\frac{1}{\Omega_{0}}\right)^{n-b-v-s+1}}\right]\right) \\
& r_{1}^{\prime}=\frac{M !}{(M-w) !(w-1) !} \sum_{k=0}^{w-2} \frac{(1-w)^{k}}{(w-2-k) !} \sum_{m=0}^{k} \frac{1}{\left(\Psi_{1} \sigma\right)^{w-1-k+m}} \sum_{v=0}^{m} \frac{(-1)^{v}}{(m-v) ! v !} \frac{\Psi_{t}^{w-1-k+v}}{(w-1-k+l)}, \quad r_{5}^{\prime}=\Psi_{t}^{m-v}\left(1-\left(\frac{v-1}{w}\right)^{w-1-k+v}\right) \\
& r_{2}^{\prime}=\sum_{j=1}^{M-w} \frac{(-1)^{j} M !}{(M-w-j) !(w-1) ! j !\left(\sigma \Psi_{1}^{w}\right)} \sum_{k=0}^{w-2} \frac{(1-w)^{k}}{(w-2-k) !} \sum_{m=0}^{k}\left(\frac{j+1}{\sigma \Psi_{1}}\right)^{m-k-1} \sum_{v=0}^{m} \frac{(-1)^{l}(w-2-k+v) !}{(m-v) ! v !}\left(\frac{-\Psi_{t}}{j}\right)^{w-1-k+v} \\
& r_{4}^{\prime}=\sum_{j=0}^{M-w} \frac{(-1)^{j} M !}{(M-w-j) !(w-1) ! j !\left(\sigma \Psi_{1}\right)^{w}} \sum_{k=0}^{w-2} \frac{(1-w)^{k}}{(w-2-k) !} \sum_{m=0}^{k} \frac{(w-2-k+m) !}{m !(w-1)^{m}}\left(\frac{j+1}{\sigma \Psi_{1}}\right)^{m-k-1}\left(\frac{(w-1) \sigma \Psi_{1}}{w+j}\right)^{w-1-k+m}, r_{25}^{\prime}=\frac{1}{n !}\left(\frac{-j \Psi_{t}}{\sigma \Psi_{1}}\right)^{n}
\end{aligned}
$$

\section{Abbreviations}

CRN: Cognitive radio network; CSI: Channel state information; ER: Eavesdropper; GSC: Generalized-selection combining; MRC: Maximal ratio combining; MS-GSC: Minimum selection GSC; PU: Primary user; SOP: Secrecy outage probability; SR: Secondary receiver; SU: Secondary user; TAS: Transmit antenna selection

\section{Acknowledgements}

This research has been supported by the Department of Science and Technology (DST) project of India (grant code no. YSS / 2015 /001738/ES).

\section{Funding}

This study was funded by the Department of Science and Technology, India, grant no. (YSS/2015/001738/ES).

\section{Authors' contributions}

Both authors examine the secrecy performance of an underlay CRN with minimum selection-generalized selection combining (MS-GSC) over Rayleigh fading environment and derived closed-form expression for exact and asymptotic secrecy outage probability. Both authors read and approved the final manuscript. 


\section{Competing interests}

The authors declare that they have no competing interests.

\section{Publisher's Note}

Springer Nature remains neutral with regard to jurisdictional claims in published maps and institutional affiliations.

\section{Author details}

${ }^{1}$ Department of Electronics and Communication Engineering, National Institute of Technology, Hamirpur, India. ${ }^{2}$ Department of Electrical Engineering, Indian Institute of Technology, Jammu, India.

Received: 5 September 2017 Accepted: 12 April 2018

Published online: 10 May 2018

\section{References}

1. N Yang, L Yeoh, M Elkashlan, Transmit antenna selection for security enhancement in MIMO wiretap channels. IEEE Trans. Commun. 61(1), 144-154 (2013)

2. M Elkashlan, L Wang, TQ Duong, et al, On the security of cognitive radio networks. IEEE Trans. Veh. Technol. 64(8), 3790-3795 (2015)

3. M Gastpar, On capacity under receive and spatial spectrum-sharing constraints. IEEE Trans. Inf. Theory. 53(2), 471-487 (2007)

4. A Goldsmith, SA Jafar, I Maric, Breaking spectrum gridlock with cognitive radios: an information theoretic perspective. Proc. IEEE. 64, 3790-3795 (2009)

5. A Mukherjee, AL Swindlehurst, Robust beamforming for security in MIMO wiretap channels with imperfect CSI. IEEE Trans. Signal Process. 59, 351-361 (2011)

6. J Huang, AL Swindlehurst, Cooperative jamming for secure communications in MIMO relay networks. IEEE Trans. Signal Process. 51, 4871-4884 (2011)

7. HV Poor, Information and inference in the wireless physical layer. IEEE Wirel. Commun. 19(1) (2012). February 2012

8. HE Fangming, M Hong, W Wang, Maximal ratio diversity combining enhanced security. IEEE Commun. Lett. 15, 509-511 (2011)

9. H Alves, RD Souza, M Debbah, Performance of transmit antenna selection physical layer security schemes. IEEE Signal Process. Lett. 19, 372-375 (2012)

10. N Yang, P Yeoh, M Elkashlan, Transmit antenna selection for security enhancement in MIMO wiretap channels. IEEE Trans. Commun. 61, 144-154 (2013)

11. J Huang, A Mukherjee, AL Swindlehurst, Secure communication via an untrusted non-regenerative relay in fading channels. IEEE Trans. Signal Process. 61, 2536-2550 (2013)

12. H Zhao, D Wang, C Tang, et al, Physical layer security of underlay cognitive radio using maximal ratio combining. Front. Inf. Technol. Electron. Eng. 17(9), 929-937 (2016)

13. H Liu, H Zhao, C Tang, et al, Physical-layer secrecy outage of spectrum sharing CR systems over fading channels. Sci. China Inf. Sci. 59(10), 102-302 (2016)

14. H Zhao, Y Tan, et al, Secrecy outage on transmit antenna selection/maximal ratio combining in MIMO cognitive radio networks. IEEE Trans. Veh. Technol. 65((12)), 6-10242 (2016)

15. H Lei, M Xu, I Ansari, et al, On secure underlay MIMO cognitive radio networks with energy harvesting and transmit antenna selection. IEEE Trans. Green Commun. Netw. 1(2), 192-203 (2017)

16. H Lei, H Zhang, IS Ansari, et al, On secrecy outage of relay selection in underlay cognitive radio networks over Nakagami- $m$ fading channels. IEEE Trans. Cogn. Commun. Netw. 3(4), 614-627 (2017)

17. G Pan, J Ye, et al, Secure hybrid VLC-RF systems with light energy harvesting. IEEE Trans. Commun. 65(10), 4348-4359 (2017)

18. C Tang, G Pan, et al, Secrecy outage analysis of underlay cognitive radio unit over Nakagami-m fading channels. IEEE Wirel. Commun. Lett. 3(6), 609-612 (2014)

19. C Zhang, G Wang, et al, Secrecy outage analysis on underlay cognitive radio system with full-duplexmsecondary user. IEEE Wirel. Commun. Lett. IEEE Access. 3(6), 25696-25705 (2017)

20. MK Simon, MS Alouini, Performance analysis of generalized selection combining with threshold test per branch (T-GSC). IEEE Trans. Veh. Technol. 51(5), 1018-1029 (2002)
21. A Annamalai, G Deora, C Tellambura, in Wireless Communications and Networking, 2003. WCNC 2003. 2003 IEEE. Unified analysis of generalized selection diversity with normalized threshold test per branch, vol. 2 (IEEE, 2003), pp. $752-756$

22. X Zhang, NC Beaulieu, in Global Telecommunications Conference, 2004. GLOBECOM'04. IEEE. Threshold-based hybrid selection/maximal-ratio combining over generalized fading channels, vol. 1 (IEEE, 2004), pp. $462-468$

23. H Lei, H Zhang, et al, Secrecy outage performance for SIMO underlay cognitive radio systems with generalized selection combining over Nakagami- $m$ channels. IEEE Trans. Veh. Technol. 65(12), 10126-10132 (2016)

24. N Yang, PL Yeoh, et al, MIMO wiretap channels: secure transmission using transmit antenna selection and receive generalized selection combining. IEEE Commun. Lett. 17(9), 1754-1757 (2013)

25. L Wang, M Elkashlan, et al, Secure transmission with antenna selection in MIMO Nakagami- $m$ fading channels. IEEE Trans. Wirel. Commun. 13(11), 6054-6067 (2014)

26. Y Deng, L Wang, M Elkashlan, et al, Generalized selection combining for cognitive relay networks over Nakagami- $m$ fading. IEEE Trans. Signal Process. 63(8), 1993-2006 (2015)

27. P Gupta, N Bansal, RK Mallik, in Communications, 2004 IEEE International Conference on. Analysis of minimum selection GSC in Rayleigh fading, vol. 6 (IEEE, 2004), pp. 3364-3368

28. HC Yang, New results on ordered statistics and analysis of minimum-selection generalized selection combining (GSC). IEEE Trans. Wirel. Commun. 5(7), 1876-1885 (2006)

29. J Lee, JG Wang, et al, Outage probability of cognitive relay networks with interference constraints. IEEE Trans. Wirel. Commun. 10(2), 390-395 (2011)

30. R Etkin, A Parekh, D Tse, Spectrum sharing for unlicensed bands. IEEE J. Sel. Areas Commun. 25(2), 517-528 (2007)

31. IS Gradshteyn, IM Ryzhik, Table of integrals, 7th ed. (Academic, San Diego, 2007)

\section{Submit your manuscript to a SpringerOpen ${ }^{\circ}$ journal and benefit from:}

- Convenient online submission

- Rigorous peer review

- Open access: articles freely available online

- High visibility within the field

- Retaining the copyright to your article

Submit your next manuscript at $\gg$ springeropen.com 